\title{
Civilisations
}

Revue internationale d'anthropologie et de sciences

humaines

39 | 1991

Japon : les enjeux du futur

\section{L'État et le monde des affaires au Japon}

\section{Philippe Debroux}

URL : http://journals.openedition.org/civilisations/1671

DOI : 10.4000/civilisations. 1671

ISSN : 2032-0442

\section{Éditeur}

Institut de sociologie de l'Université Libre de Bruxelles

\section{Édition imprimée}

Date de publication : 30 octobre 1991

Pagination : 445-469

ISBN : 2-87263-044-9

ISSN : 0009-8140

\section{Référence électronique}

Philippe Debroux, «L'État et le monde des affaires au Japon », Civilisations [En ligne], 39 | 1991, mis en ligne le 06 juillet 2009, consulté le 30 avril 2019. URL : http://journals.openedition.org/ civilisations/1671; DOI : 10.4000/civilisations.1671

\section{(c) Tous droits réservés}


L'ETAT ET LE MONDE DES AFFAIRES AU JAPON

Philippe DEBROUX

\section{Introduction}

L'Etat japonais passe pour l'un des plus interventionnistes et centralisés du monde industriel. Cependant, il est aussi un de ceux où les firmes et marchés des capitaux privés jouent le rôle le plus important par rapport aux firmes et investissements publics.

Malgré des contraintes sévères d'approvisionnement en matières premières, l'Etat japonais n'a jamais développé de champion national pétrolier pouvant rivaliser avec les grandes multinationales anglo-saxonnes. Il n'a pas non plus de producteurs d'énergie électrique publics.

En dépit d'une politique industrielle active, le Japon n'a plus eu de grandes firmes publiques producteurs de biens et services depuis les privatisations du début de l'ère Meiji.

Si des institutions financières telles que la Japan Development Bank ou l'Exim Bank jouent un rôle important dans le commerce extérieur et l'allocation des fonds aux pays en voie de développement, ce sont les banques privées qui ont mené la politique active de crédit qui a permis aux firmes japonaises de maintenir un haut niveau d'investissement après la seconde guerre mondiale.

Dans les économies mixtes de l'Europe occidentale, l'Etat est encore souvent un producteur soit rival, soit partenaire de l'industrie privée. L'Etat japonais n'est ni l'un ni l'autre. Au contraire de pays comme la France, la Grande-Bretagne et 
même l'Italie, il n'y a quasiment pas d'exemple de combinaisons d'éléments stratégiques et politiques qui ont conduit l'Etat japonais à imposer une présence concurrentielle sur le marché. Porté par la vague mondiale de privatisation, le gouvernement japonais a même entamé le processus de cession de ses parts dans les quelques activités grâce auxquelles il avait une présence commerciale sur le marché : sel, tabac, chemin de fer et télécommunication.

Il ne faudrait pas croire cependant que la quasi absence de propriété publique commerciale signifie que l'Etat japonais a accepté spontanément de privilégier le contrôle réglementaire. L'image du MITI (Ministère du Commerce International et de l'Industrie) omnipotent et omniscient traverse tout de suite les esprits. Ses fonctionnaires d'élite seraient censés savoir qu'ils ne doivent intervenir qu'avec modération pour ne pas causer de distorsions des marchés. Une politique de conformité au marché serait préférable, le marché étant un instrument de la politique qu'on peut efficacement contrôler par des moyens indirects plutôt qu'en s'opposant directement à ses règles. En ce sens on pourrait penser que le fait que l'Etat soit un acteur commercial actif ou non importe finalement peu. La réglementation donne une plus grande flexibilité sans compromettre l'autorité de l'Etat. Celui-ci peut contrôler sans avoir la propriété et si le besoin s'en fait sentir, il peut l'acquérir sans difficulté.

En fait, il n'en est pas ainsi. Si on examine le passé, l'intervention directe de l'Etat a toujours été importante dans tous les domaines précités, que ce soit celui de l'énergie ou celui de la production de biens. Dans le Japon dictatorial d'avant guerre comme dans celui devenus démocratique d'après guerre, il a toujours cherché à imposer ses vues aux intérêts privés, si possible par une intervention directe sur le marché. Clairement, la réglementation a toujours été un pis aller pour les concepteurs de la politique industrielle japonaise car, quelle que 
soit son domaine d'application, elle les fait dépendre de la coopération et des préférences des acteurs privés.

L'Etat japonais a joué et joue encore à contre-coeur le jeu de la conformité au marché parce qu'il a trouvé sur sa route des intérêts privés puissants, relativement solidaires et stables. $\mathrm{Au}$ cours du processus de développement du commerce et de l'industrie, les dirigeants des grandes entreprises et des institutions financières ont pu établir des alliances durables avec les politiciens et représentants de l'administration. L'interpénétration des relations au sein du triangle politiqueadministration-entreprises, a permis une participation active de ces mêmes intérêts privés à l'élaboration et à la mise en oeuvre de la réglementation. Par cela, ils ont pu aussi contrôler les possibles enfreintes aux règles du marché qu'aurait voulu commettre les pouvoirs publics.

C'est en ce sens qu'on peut dire que l'intervention de l'Etat japonais peut paraitre paradoxale. Sa présence est massive et visible dans tous les secteurs de la vie écoomique mais elle l'est parce que justement les acteurs privés via leurs associations professionnelles, leur participation aux commissions mixtes, les possibilités de carrière et les incitants qu'ils offrent aux membres de l'administration et au personnel politique, ont appris comment à la fois contenir, limiter mais aussi soutenir la puissance de l'Etat aux moments opportuns.

Il va sans dire que le marché et les structures de l'Etat sont tout autant les produits de la négociation et de l'intérêt du moment que dans les autres démocraties industrielles. Les intérêts privés s'accommodent d'un tel Etat en lui reconnaissant le pouvoir de réglementation tout en gardant le contrôle du marché. L'Etat japonais, lorsqu'il décide d'intervenir, s'efforce d'aménager des structures de marché en état de changement permanent en faisant participer les acteurs privés à 
l'élaboration et la mise en oeuvre de la règlementation. En faisant cela, il renforce systématiquement la position des firmes existantes. Ces actions conformes au jeu du marché n'arrivent jamais à empêcher des transformations de la structure de celui-ci qui est naturellement en évolution.

Ce sont ces changements mêmes qui permettent d'expliquer la présence constante et massive de l'Etat dans l'économie. Parce qu'il n'y a pas de fin à l'instabilité du marché, il n'y en a pas non plus aux négociations permanentes entre l'Etat et le privé pour en orchestrer les mouvements. Il est difficile de parler dans ces conditions de domination bureaucratique ou de capitalisme d'état. On pourrait plutôt parler d'un cadre d'acceptation réciproque et provisoire. Certes, l'Etat aide souvent à structurer les tendances du marché. S'il n'est pas présent institutionnellement sur celui-ci, il dispose d'un certain nombre de points d'accès lui permettant d'observer et de conserver une flexibilité d'intervention à tout moment tout en se tenant en retrait. Cependant, les choix finaux sont toujours structurés par le processus sans fin de négociation avec les intérêts privés.

\section{Autonomie et capacité de l'Etat}

Beaucoup d'études ont été réalisées sur l'évaluation de la manière et de l'efficacité avec lesquelles les états interviennent dans leurs économies pour restructurer les marchés. En partageant le principe que l'Etat peut agir de manière autonome et que cela se fera d'une manière prévisible, la plupart d'entre elles, sont moins concernées par son autonomie relative que par la relativité de sa capacité.

Pour essayer de différencier "Etats faibles" et "Etats forts" et en parlant dans tous les cas d'une institution cohérente, identifiable et autonome, on doit lier la capacité de l'Etat au 
caractère du processus d'élaboration de la politique tel qu'il se reflète dans l'équilibre historique des forces entre l'Etat et la société. L'Etat le plus fort peut changer le comportement des acteurs privés aussi bien que la structure économique elle-même alors qu'un autre plus faible peut résister aux exigences privées mais n'est pas en mesure de changer leurs préférences. Quant au plus faible, il ne peut même pas résister à ces exigences privées et tombe sous leur domination. L'Etat japonais est souvent associé à la France comme archétypes de l'Etat "fort" au sein des démocraties industrielles. Tous deux sont supposés pouvoir influencer les marchés grâce à un appareil administratif très centralisé, largement isolé de la société civile et de la pression politique parlementaire. Leur prestige leur permet de recruter l'élite des meilleures universités ou Grandes Ecoles. Le pouvoir de leur bureaucratie a été renforcé depuis la guerre par le maintien au pouvoir pendant plus de 30 ans de majorités conservatrices stables, les 10 ans d'alternance socialiste en France n'ont rien changé à cet égard.

A l'autre extrême, nous avons les Etats-Unis et l'Italie comme archétypes de l'Etat "faible" souffrant tous deux de la faiblesse de leur organisation administrative et de la grande fragmentation des intérêts en présence. A priori, ces deux Etats sont moins capables d'arbitrer les conflits politiques et la concurrence pour l'attribution des ressources de l'Etat. Ils manquent donc de la capacité de transformation de la société et de résistance aux pressions privées.

L'acceptation du principe que les Etats peuvent agir de manière autonome invite à poser un certain nombre de questions concrètes sur cette capacité de l'Etat. Tout d'abord, on doit chercher à savoir si elle est cohérente dans tous les secteurs et dans le temps. Ensuite, il faut se demander si elle permet une prévision du choix de la politique qui sera suivie. En effet, dans un Etat "fort" comme la France, il est arrivé que des intérêts 
privés parviennent à le contrôler de manière effective : c'est le cas du lobby agricole et du nucléaire. D'un autre côté, il y a beaucoup de cas d'interventions d'Etats "faibles" qui ont eu des implications importantes dans le développement économique national : c'est le cas notamment du New Deal de Franklin Roosevelt et de l'action d'Enrico Mattei à la tête de l'IRI en Italie.

Dans le cas de la France et du Japon, les gouvernements conservateurs en place et contrôlant l'administration ont tout d'abord cherché à s'associer à la grande industrie et aux milieux financiers en marginalisant la gauche et les associations de travailleurs la soutenant. Leur objectif était le passage de sociétés agraires à l'industrie : cela s'est fait par l'encouragement et la promotion des secteurs à haute valeur ajoutée, une politique d'augmentation des salaires dans l'industrie et la création d'avantages pour leurs firmes sur les marchés internationaux. La transformation a été systématiquement facilitée par l'utilisation de la politique de crédit, du système fiscal et de la politique commerciale. Cependant, pour les autorités japonaises, il ne s'est jamais agi de créer un système de subsidiation à long terme mais d'aider au développement de secteurs bénéficiant d'avantages comparatifs au niveau international. L'augmentation de la production et la modernisation de l'appareil productif avec l'aide publique n'assure pas en soi la compétitivité internationale. Le bénéfice de l'expansion dépend des choix technologiques et de l'organisation de la production par les firmes elles-mêmes. Alors qu'au Japon, l'administration a finalement adopté des politiques de conformité au marché après négociation, en France, l'Etat a cherché à supplanter le marché en abritant de la concurrence les firmes et secteurs sélectionnés et en promouvant des projets ne s'insérant pas dans des plans commerciaux. 
La domination de l'industrie par le MITI en tant que soutien de la politique d'expansion économique avec une vision sur les structures adéquates de secteurs spécifiques a continué aussi longtemps que cette expansion générait des possibilités de croissance. Les gains étaient mesurés en termes de baisse des coûts de production, de prix sur le marché intérieur et à l'exportation et de parts de marché sur les marchés étrangers. Le MITI dictait les termes d'accès au marché japonais et pouvait donc fournir aux firmes un marché stable sans concurrence étrangère sur lequel pouvait se bâtir un réseau de production et de distribution concurrentiel.

En général, les coalitions politiques soutenant une intervention étatique et même la propriété publique ont tendance à se grouper autour des secteurs dominés par des intérêts étrangers. Comme les producteurs nationaux étaient protégés, l'Etat n'a pas ressenti le besoin de contrôler directement en tout ou en partie certains secteurs stratégiques de l'économie interne. Cela aurait augmenté les perspectives d'interventions directes pouvant entraîner une distorsion du marché. Au lieu de créer une présence étatique concurrentielle sur le marché, le gouvernement a encouragé activement la concurrence interne en accordant un large soutien aux firmes en expansion. Il a donné un accès au financement pour l'investissement, a accordé des déductions fiscales assurant un cash flow suffisant pour maintenir la liquidité, un soutien en recherche et développement et une aide de promotion à l'exportation.

Ces politiques publiques ont changé les options stragégiques des entreprises. Une expansion aussi rapide des secteurs de l'acier et de l'automobile exigeant de très grands investissements initiaux devait être financée par la dette. Jamais ni les entreprises ni les banques n'auraient pu la gérer sans facilités fiscales ni diffusion du risque d'endettement. 
Quand la base de production fut reconstruite et que les produits manufacturés japonais ont commencé à jouir d'un avantage concurrentiel, cette domination prit fin. Les entreprises se sont rendues plus indépendantes et, comme on le verra plus loin, se sont opposées de plus en plus fréquemment à l'interventionnisme gouvernemental. Celui-ci n'a pas pris fin mais le MITI a perdu l'initiative. Dans les secteurs de haute technologie comme les télécommunications ou l'électronique, il a commencé à coordonner de grands projets initiés non plus par lui mais par les firmes privés, tout en gérant des situations de transition comme dans les secteurs de la construction navale, de la pétrochimie ou de la production d'aluminium.

Ces faits montrent que même un Etat dit "fort" a toujours finalement reflété les exigences du monde des affaires.

Par conséquent, une conception indifférenciée n'est pas suffisante pour prédire la nature, la mesure et l'efficacité de l'intervention de tels Etats. Tous ces éléments varient historiquement et de secteur à secteur. Même les Etats dits faibles interviennent quand des ajustements au marché sont requis et là où les acteurs privés n'ont pas encore pu fixer leur choix de marché ou de technologie. De plus, ces choix sont établis politiquement et dépendent en grande partie des différentes manières avec lesquelles les intérêts divergents s'organisent dans leurs relations avec l'Etat.

Pour expliquer les nombreux procédés utilisés par l'Etat pour discerner entre des firmes ou des secteurs et orienter les équilibres dans un sens ou un autre, on peut dire que la capacité de l'Etat en elle-même est sans doute moins importante que les coalitions et conflits déterminés historiquement. Depuis les arrangements entre le gouvernement, les grands propriétaires terriens et les industriels de la fin du 19ème siècle, les partis conservateurs japonais ont joué un rôle de charnière entre ces 
secteurs clés. L'Etat japonais de l'ère Meiji (1868) s'est basé sur l'expérience gouvernementale et constitutionnelle prussienne en créant une machine étatique forte et centralisée. L'élaboration de la politique et le contrôle de la société se trouvait entre les mains d'une classe bureaucratique. Les pouvoirs du Parlement et des partis politiques étaient volontairement faibles et les pouvoirs locaux sous la surveillance vigilante de l'Etat central. Les droits sociaux et politiques des citoyens avaient volontairement moins de poids que leurs devoirs et obligations vis-à-vis l'un de l'autre et de l'Etat. Les conflits et compromis n'étaient pas considérés comme des conséquences logiques de complexités sociales ou d'intérêts en état de concurrence légitime. Bien au contraire, ils représentaient des menaces d'intérêts égoistes et intolérables pour l'harmonie sociale vue comme essentielle pour la souveraineté nationale, le développement du pays et le bien-être.

Après la guerre, les droits des citoyens ont été reconnus et le Parlement est constitutionnellement le plus haut organe de l'Etat. L'administration est maintenant celle de toute la communauté et non plus celle de l'Etat. Cependant, les forces d'occupation alliées (américaines) ont par la force des choses été obligées de s'appuyer sur cette même administration dans les années de troubles et d'instabilité sociale et politique des années d'après guerre. Il n'y a pas eu d'effort important pour en réduire les pouvoirs, ce qui fait que de nos jours l'administration japonaise jouit probablement encore de prérogatives plus importantes que dans les autres démocraties parlementaires. Après la fusion des 3 partis conservateurs en 1955 pour constituer le Parti Libéral Démocrate, celui-ci est devenu un parti de gouvernement incontournable. Il domine la scène plus encore que la sociale démocratie italienne car il n'a jamais dû s'appuyer sur d'autres forces pour constituer une majorité. Il a investi tous les rouages de l'administration, multiplié les flux 
entre celle-ci, le monde politique et celui des affaires. Les hauts fonctionnaires dépendent de lui pour leur nomination et promotion. Il a fait de même avec les entreprises en créant des comités de concertation permanents et en favorisant les va et vient de cadres supérieurs et moyens entre le privé et l'administration. Ceci a assuré la continuité et la cohérence de la politique japonaise après la guerre.

Nous devons alors nous demander comment les relations entre le passé et le présent peuvent être précisées. L'accumulation historique d'engagements et de luttes politiques antérieures définit au mieux ce qui est ou n'est pas possible. Les structures politiques et administratives sont liées à des phases de développement dans des environnements nationaux particuliers. Cela signifie que l'identité, l'autonomie et la capacité des Etats varient suivant le mode de développement national.

Aux Etats-Unis, l'industrie est arrivée à maturité avant l'Etat. Or, un Etat faiblement institutionnalisé ne peut suppléer à sa faiblesse qu'en renforçant la réglementation, qui est l'une des seules armes qu'il a. Cette large présence de la réglementation est devenue aux Etats-Unis une partie de la tradition administrative identifiée avec un Etat faible.

Il n'a jamais existé au Japon de tradition d'esprit d'entreprise autonome de l'Etat et menant à des politiques différent fondamentalement de la sienne comme en Grande-Bretagne ou aux Etats-Unis. D'un autre côté, les milieux industriels n'ont jamais eu à faire à un Etat soumis à des groupes de pression représentant des intérêts industriels archaïques empêchant le passage à la grande industrie comme dans la France de la 3ème et de la 4ème républiques. 
Le Japon est passé directement d'une économie féodale agraire à une économie industrielle modérément planifiée. La concurrence entre firmes est intense mais cette concurrence est limitée et dirigée par les actions de l'Etat et les efforts de collaboration des firmes et des banques. L'administration ne contrôle pas le marché. Elle contribue consciemment au développement de secteurs particuliers et aide à établir les conditions d'investissement et de risque pour promouvoir le développement à long terme et la compétitivité internationale. Le MITI a été et est toujours dans une certaine mesure un acteur avec ses propres buts et moyens d'intervenir sur le marché pour les atteindre. Le gouvernement ne fait pas seulement usage des forces concurrentielles se produisant naturellement sur le marché mais il induit souvent la concurrence qu'il dirige. En faisant cela, il crée le marché pour les produits et les conditions de profit, ce qui favorise l'entrée de nouveaux concurrents. Cela se termine souvent par la création de cartels qui se font et se défont mais fonctionnent et comptent que ce soit dans la haute technologie maintenant ou dans l'acier il $y$ a 20 ans.

La structure du monde des affaires soutient cet accord de concurrence contrôlée. Au Japon, les firmes privées ont toujours encouragé le type d'intervention étatique leur permettant de limiter légalement la production, d'allouer des marchés, de contrôler les prix et de se partager les ventes. Les luttes pour le contrôle étatique et l'autonomie avant la 2ème guerre mondiale ont été résolues par la création de cartels légaux garantissant la propriété privée et l'indépendance de la gestion. Cette solution a profondément influencé l'évolution de l'économie japonaise et de ses institutions, particulièrement les limites de l'Etat en tant que participant commercial en définissant ce qu'un Etat capitaliste est et devrait être. 


\section{Termes de l'acceptation réciproque}

Le type d'intervention de l'Etat dans l'économie dépend entre autre de la nature de la coalition au pouvoir. Elle sera probablement en conformité avec les règles du marché là où les coalitions au pouvoir ont une large base et la politique d'acceptation réciproque est stable. Il n'y a pas de barrières ni d'équilibre permanent de pouvoir entre l'Etat et la société mais un processus de réassurance mutuelle entre les intérêts privés et les autorités publiques. Le processus fonctionne d'autant mieux que les parties à ces négociations sont stables ainsi que les institutions.

La politique japonaise contemporaine a été dominée par une alliance entre des secteurs clés qui ont montré une capacité persistante à définir l'agenda politique national et fixer des priorités politiques pour le pays par une négociation permanente. La juridiction publique et privée sur les marchés n'est pas monopolisée par l'Etat japonais ou par les firmes privées mais elle est négociée, ce qui veut dire qu'il y a une interdépendance entre le pouvoir public et le privé. De même, le contrôle, défini en termes de leadership et d'autorité n'est pas attribué d'office. Les termes de l'accord mutuel ne sont pas les mêmes sur tous les marchés et donc la distribution du pouvoir y est aussi différente. Dans tous les cas, les deux parties concourrent pour définir leur propre vision de l'intérêt national en s'efforçant toujours de transférer les coûts y afférant à l'autre, tout en visant le profit maximum.

Les conflits sur le contrôle des ressources productives et naturelles et les frictions en sens divers menant à un accord réciproque définissent la nature et la dimension des interventions directes de l'Etat dans les démocraties industrielles. De tous temps et en tous lieux, les intérêts privés ont lutté pour séparer l'aide de l'Etat du contrôle. Les 
constructeurs automobiles ont bénéficié immensément des efforts du MITI pour restructurer le secteur des pièces détachées avec des fonds publics mais ont toujours lutté pied à pied pour conserver leur indépendance. La décision du groupe Mitsubishi en 1969 d'entrer à son tour dans ce secteur malgré l'opposition du MITI est d'ailleurs considérée comme une défaite majeure de celui-ci, marquant le début de son relatif déclin. Depuis plus de 50 ans, le gouvernement japonais a entrepris vainement d'innombrables tentatives pour intégrer verticalement le secteur pétrolier. Les grandes banques commerciales sont toujours restés sourds aux appels de fonds. Elles ont encore profité de la fragmentation de l'industrie pétrolière japonaise pendant la période englobant les deux chocs pétroliers. Ce n'est que lorsqu'elles ont jugé la profitabilité de cette situation épuisée qu'elles ont commencé à bouger au milieu des années 80 et ont favorisé la consolidation. L'Etat japonais a joué un rôle dans tout le processus mais celuici a presque toujours été précédé par de larges accords avec les intérêts privés, principalement les banques. Malgré les efforts de l'administration, le secteur ne pouvait être consolidé que lorsque les banques étaient prêtes.

Dans la plupart des pays européens, l'Etat a depuis longtemps pénétré sur le marché pour remplir des objectifs politico-socio-économiques que les firmes privées ne pouvaient remplir pour des raisons de risque ou de rentabilité. De même, une intervention a toujours semblé légitime pour corriger une stagnation sectorielle ou régionale, protéger une industrie naissante, maintenir l'emploi, pourvoir à la disponibilité de biens collectifs ou finalement socialiser des risques privés.

Une économie ou un secteur en voie de développement requiert d'autres instruments de pouvoir étatique que ceux d'un pays industrialisé. Le faible niveau d'épargne privée disponible pour l'investissement productif en France et en Allemagne peut 
expliquer historiquement l'existence de banques d'investissement publiques dans ces deux pays à la différence de la Grande-Bretagne. Sans protection étatique et capital public, la Grande-Bretagne et la France n'auraient jamais pu développer une industrie pétrolière viable. En Italie, quand les intérêts privés abandonnèrent le projet de construction de raffineries, c'est l'Etat Mussolinien qui pris le relais.

Le capital étatique a été important au Japon dans les premiers stades de développement de secteurs tels que ceux de l'acier, du chemin de fer, des armes, de l'automobile et de l'extraction minière. Bien que le système financier japonais structure les choix de l'industrie et accorde un pouvoir à l'Etat vu la position prééminente de la Banque du Japon, l'intervention publique s'est passée dans un processus politique au cours duquel le capital privé avait déjà pris le bénéfice, socialisé les risques et parcellisé la dette.

Cela a été particulièrement évident dans le cas de l'industrie charbonnière. Les investissements publics ont été primordiaux dans les premiers stades de développement minier au Japon. Ils ont ensuite décliné rapidement pour faire place à l'exploitation par les grands holdings (Zaibatsu). Au début du déclin, à l'intervention publique directe, s'en est substituée une de garant financier des risques encourus par les investisseurs privés. Lorsque dans les années 60 , le niveau de compétitivité des mines a entraîné une augmentation rapide des pertes d'exploitation et du niveau d'endettement, les intérêts privés ont négocié leur sortie avec le MITI. Celui-ci ainsi que les politiciens concernés, ont négocié la substitution de dettes publiques aux dettes privées dans leur vaine ambition de sauver une partie du secteur. En agissant de cette façon, l'Etat jouait son rôle de soutien du bien public sans menacer directement l'accumulation privée. 
Aux Etats-Unis, l'Etat n'est quasiment jamais en concurrence avec des firmes privées sur des marchés commerciaux. Cependant, les garanties financières publiques pour l'octroi de crédit hypothécaire, le payement des droits d'inscription dans l'enseignement, l'aide aux petites et moyennes entreprises et aux multinationales défaillantes, constituent une part importante du budget fédéral.

Les pouvoirs d'allocation des institutions de crédit publiques au Japon sont encore plus étendus, en partie sans doute parce qu'ils sont négociés plus largement avec des firmes privées. Ils ont un objectif commercial plus évident. De plus, les exigences d'une industrialisation tardive comme c'est le cas du Japon mènent souvent au développement de grandes banques d'investissement et à un rôle explicite de l'Etat. Les institutions financières gouvernementales offrant des crédits garantis à taux d'intérêts très bas sont beaucoup plus nombreuses que les quelques rares entreprises publiques encore actives dans tous les secteurs. Malgré la libéralisation de la fin des années 80 , le système financier japonais reste basé sur le crédit avec un contrôle sur les taux exercé par le Ministère des Finances. Ce système est un élément fondamental de toute stratégie d'ajustement menée par l'Etat par laquelle le gouvernement s'efforce consciemment de modeler des secteurs particuliers. Les choix entre les instruments commerciaux et financiers sont liés. Dans beaucoup de cas, aux Etats-Unis et au Japon, l'Etat utilise des interventions financières là et quand il ne parvient pas à établir une présence directe sur le marché ou est bloqué par des groupes nationaux ou étrangers poursuivant leurs propres stratégies de marché. Si l'allocation de crédit est sans conteste un outil efficace pour un Etat fort, l'Etat créditeur ou garant est sans doute le moins menaçant et donc le plus acceptable pour des intérêts privés. 
Le gouvernement japonais a identifié le besoin de création de banques à long terme spécialisées au début des années 50 pour diminuer la pression sur les banques commerciales en vue de l'octroi de prêts à long terme. Il existe 12 institutions financières publiques établies pour financer des objectifs spécifiques jugés d'intérêt national. Leur statut leur interdit formellement d'entrer en concurrence avec les banques commerciales et autres institutions financières. Dans ce cas aussi, le rôle gouvernemental a été précédé par une grande compréhension et considération pour les intérêts privés, particulièrement les banques. L'Etat japonais n'entre pas en concurrence ni ne détermine l'allocation de fonds par les banques privées. Les institutions financières étatiques ne sont pas seulement des pourvoyeurs additionnels de fonds. Elles sont les garants protégeant non seulement les secteurs industriels stratégiques mais les banques elles-mêmes.

La position des groupements d'intérêts vis-à-vis de l'Etat est certainement une variable importante pour déterminer leur capacité de réaliser leurs objectifs. La capacité de l'Etat et le pouvoir des intérêts privés dépendent à la fois de la centralisation des ressources et de celle des intérêts. La diversification de ceux-ci invite à une intervention de l'Etat. Quand les acteurs sur le marché sont divisés ou désorganisés, il faut s'attendre à ce que l'Etat soit en mesure de provoquer des distorsions sur le marché commercial ou financier et d'agir comme rival ou créancier de firmes privées.

Le monde des affaires n'est pas un secteur unifié au Japon comme il ne l'est d'ailleurs nulle part. Une distinction fondamentale dans l'orientation et l'influence est la taille des entreprises. Le mode général d'organisation industrielle et d'interrelations entre firmes est celui d'une large base de petites et moyennes entreprises liées collectivement et hiérarchiquement à un nombre limité de grandes entreprises. 
Ces grandes firmes sont souvent associées par des participations croisées au capital. Le petit nombre de grandes firmes joue un rôle fondamental dans l'élaboration de la politique économique alors que les plus petites firmes en subissent les conséquences sans y avoir participé.

Les quelques 600 associations commerciales et industrielles sectorielles entrent en relation directe officielle avec les agences gouvernementales. Dominées par les plus grandes firmes, elles fonctionnent comme canaux à deux voies entre leurs membres et les autorités publiques. De plus, environ 100 des grandes associations commerciales et 750 des plus grandes entreprises sont organisées dans le Keidanren (Federation of Economic Organizations) et dialoguent avec les secteurs clés de l'industrie et le gouvernement. Un grand nombre de comités fonctionnels sont en relation constante avec l'administration. Deux autres associations sont aussi très influentes dans l'élaboration de la politique macro-économique : le Keizai Doyukai (Japan Committee for Economic Development) et le Nikkeiren (Federation of Employers Associations), cette dernière étant aussi chargée des relations avec les syndicats.

En plus des services à leurs membres et de la transmission d'informations aux agences gouvernementales, elles sont aussi des conduits assurant l'observation par l'industrie de la politique gouvernementale. Les milieux d'affaires jouissent de privilèges et de leur inclusion systématique dans le processus de formulation de la politique. Ils ont accès aux biens publics et jouissent de droits d'autoréglementation. En contre partie, les industries acceptent que l'Etat ait un droit de réglementation sur les structures de marché. Les intérêts privés sont invités à participer au processus interne du gouvernement alors que l'administration participe à celui du marché. Un niveau d'autonomie de l'Etat est admis sans que soient ignorées les stratégies privées. Cela est intégré dans la guidance 
administrative du MITI qui est le mécanisme informel souvent mis en avant pour exemplifier le contrôle étatique au Japon. Les parties elles-mêmes ont la tâche de déterminer les détails de la réglementation et même parfois son domaine et niveau d'application. Il s'agit bien là d'une régulation de l'allocation des profits et changes de la réglementation. En contrepartie, les industriels acceptent que l'Etat ait un droit de juridiction sur les structures de marché. Les intérêts privés sont invités à participer au processus interne du gouvernement alors que l'administration participe à celui du marché. Un niveau d'autonomie de l'Etat est admis sans que soient ignorées les stratégies privées.

En ce qui concerne le secteur financier, la situation de pays à pays conditionne la capacité relative de négociation : l'Allemagne connait un système dominé par de très grandes banques, extrêmement jalouses de leur indépendance vis-à-vis de l'Etat; les Etats-Unis ont un système très déconcentré avec un éparpillement des intérêts jouant en défaveur des intérêts privés; le Japon a un système dominé longtemps par la dépendance des grandes banques privées vis-à-vis de la Banque du Japon et donc du Ministère des Finances pour l'accès aux fonds destinés à l'industrie. Les banques japonaises sont puissantes certes mais jamais le gouvernement n'a permis que se créent les Behemoths dominant le monde financier allemand.

En ce qui concerne le degré de centralisation de l'Etat, la présence d'un large consensus et accord dans la coalition conservatrice, ne doit pas nous faire négliger l'importance des divisions qui existent parmi les membres de cette coalition. Celle-ci est composée d'acteurs multiples avec leurs intérêts particuliers et leurs perspectives, parfois irréconciliables. L'orientation du Ministère des Affaires Etrangères d'amélioration des relations avec les autres pays en a fait un avocat de l'ouverture de l'économie; les liens du MITI avec la 
grande industrie lui font veiller à ce que cette orientation ne heurte pas les intérêts industriels locaux. Le Ministère des Finances, à cause de ses liens avec les banques et ses soucis d'orthodoxie fiscale cherche à maintenir l'équilibre entre ce qui est désirable à l'intérieur et à l'extérieur et ce qui est fiscalement possible.

Les disputes entre le MITI et le Ministère des Finances ont significativement affecté l'intervention de l'Etat dans la création de la New Energy Development Organization en vue de consolider le secteur pétrolier et tous les projets de rationalisation du secteur charbonnier de 1955 à 1968.

La volonté du MITI de se concentrer sur les secteurs à haute intensité en capital et sur la haute technologie s'est heurtée de front à l'opinion de la Banque du Japon suivant laquelle une concentration sur des secteurs à haute intensité de main-d'oeuvre semblait plus appropriée pour une économie ayant peu de ressources naturelles et de capital. Les secteurs sélectionnés par le MITI semblaient les moins appropriés pour le Japon suivant la théorie des coûts comparatifs.

Ceci montre que le processus qui génère la présence de l'Etat sur les marchés est segmenté aussi bien au niveau de l'Etat que par le marché. Le MITI n'est certainement plus le seul coordonnateur de la politique économique ni un arbitre impartial des conflits d'intérêts.

\section{Conclusion}

Le Japon est sans doute un cas particulier pour ce qui est de l'analyse des relations état-industrie dans les démocraties industrielles. En Occident, les approches libérales et marxistes de l'Etat et de la société partagent la croyance fondamentale que les préférences de la société prévalent quand elles divergent 
des préférences de l'état. Le Japon, au contraire, a une longue tradition explicite de l'Etat dans l'explication du développement économique. Donc, à première vue, le Japon semble le cas le moins ambigu d'autonomie et de puissance étatique. En considérant l'autonomie relative de l'Etat, l'indépendance et le contrôle étatique sont placés en premier lieu sans séparer soigneusement indépendance d'interdépendance et contrôle de juridiction.

En évaluant la capacité de l'Etat, la vision dominante sur le Japon est celle de puissance et souvent de capacité de vision alors que l'Etat est soumis à un nombre élevé de contraintes et a un don de vision qui a toujours laissés sceptiques les industriels japonais.

Les choix qui ont présidé au développement du Japon n'ont jamais eu le caractère d'inexorabilité qu'on leur prête. Ils ont généralement été changeant et soumis aux contingences. L'intervention dans le secteur de l'acier a permis de préserver le marché intérieur pour les firmes japonaises, de fournir les fonds d'investissement et de gérer l'investissement pour éviter la surproduction. Cependant, en même temps, le gouvernement a été incapable de dicter le sort des fabricants textiles, des assembleurs automobiles et des firmes électroniques.

Les grandes ressources financières des fabricants automobiles leur ont permis de se libérer des diktats du gouvernement et de refuser tout regroupement. Sony qui a été un pionnier pour l'introduction de composants miniaturisés dans l'électronique de consommation est toujours resté en dehors des accords gouvernementaux. Les fabricants du textile étaient voués à un déclin inexorable par le MITI mais ils ont prospéré. 
Le Japan Inc. n'a jamais existé, s'il s'agissait de décrire un système dans lequel les élites de l'industrie, de l'administration et de la politique, toutes naturellement concurrentes, parvenaient à un accord global pour le bien suprême du pays à travers un réseau entremêlé de groupes et d'institutions de consultation et de négociation.

La plupart des travaux effectués sur le Japon insistent sur les pouvoirs de l'Etat et pas du tout sur les contraintes s'exerçant sur lui. Le rôle de l'Etat japonais ne ressemble pas nécessairement à celui de la France ou des Etats-Unis. Cette distinction a une signification allant au-delà d'une meilleure compréhension des relations industrie-état au Japon. L'industrialisation tardive du Japon et ses succès économiques ont renforcé l'idée qu'il n'y a qu'une seule rationalité économique, celle d'une machine standardisée, efficace et centralisée. En cela, le Japon est devenu le porteparole ou le symbole de la modernité économique. Or, on peut démontrer qu'une grande variété de possibilités subsistent dans les relations état-marché. Aucun leadership a priori de l'état ne doit être supposé même dans les états au développement tardif ni dans ceux dont l'homogénéité culturelle pourrait faire croire que les conditions d'un leadership autoritaire de l'Etat est probable. En fait, l'Etat moteur du développement ne doit pas nécessairement être un Etat fort.

L'Etat japonais est omniprésent sur le marché mais il n'a pas de rôle de guide ou de superviseur. Les capitalistes japonais ont toujours lutté avec acharnement au cours des périodes de haute croissance comme pendant les guerres et les dépressions pour séparer l'aide de l'Etat de son contrôle. Dans aucun cas, ils n'ont privilégié le patriotisme par rapport au profit. Même pendant la guerre, les intérêts industriels ont été réticents à céder aux exigences des militaires. 
Au Japon, a toujours persisté un équilibre délicat de la juridiction étatique combinée au contrôle privé. Aucune interprétation monolythique ne donne de solution à la question. L'état et les acteurs privés s'affrontent souvent avec des conséquences importantes. Au sein de la bureaucratie, des luttes internes féroces ont toujours eu lieu sans parler de la concurrence entre firmes privées sur le marché.

Toute identification claire de l'intérêt de l'état et du secteur privé doit par conséquent être limitée par une appréciation de la manière dont cet intérêt est structuré et de la mesure de l'interpénétration. L'état japonais a agi comme un acteur autonome avec une position stratégique dans des sphères importantes telles que l'élaboration d'une politique de l'accès ou de l'énergie ou bien de contrôle des institutions financières privées quand il s'est agi de négocier l'attribution de marchés. Cependant ce rôle stratégique a pu être rempli parce que les acteurs étatiques ont accepté de négocier leur autorité, de s'allier avec des acteurs privés dont les demandes étaient conformes à leur conception de l'ajustement économique. Donc, on peut dire que l'autonomie de l'Etat a toujours été confortée par des intérêts privés "alliés" avec lesquels la négociation est perpétuelle.

Dans les années 30, les fonctionnaires en charge de l'économie suggéraient que pour atteindre la plus grande efficacité de l'investissement en capital, il était nécessaire de procéder à des fusions, d'encourager les accords commerciaux entre entreprises concernant le niveau de production et la division des marchés en vue d'empêcher une "concurrence excessive".

Les années 80 ont vu apparaître le même souci des milieux officiels et privés d'éviter une surconcurrence dans des secteurs tels que la construction navale, la pétrochimie ou la production 
de métaux. Il n'y a jamais eu au Japon, au contraire des Etats-Unis, d'hostilité vis- à-vis des monopoles privés. Alors que l'Etat américain accordait la priorité au contrôle des trusts, l'Etat japonais collaborait avec eux pour les aider à se développer. Si la société industrielle japonaise est très concurrentielle, ni les milieux publics ni les privés n'ont jamais cru que les marchés permettent naturellement un ajustement dans le meilleur intérêt du capital et des citoyens.

En ce sens, l'Etat japonais ressemble à l'Etat français par ce manque de confiance dans le marché. C'est là l'ironie de la situation : un état ne croyant pas au marché, créant et manipulant des intérêts mais supervisant des interventions reproduisant sans cesse de nouvelles structures de marché et des acteurs privés jaloux de leur autonomie mais infiltrant l'Etat et l'aidant constamment à développer et mettre en oeuvre son outil réglementaire.

En fait, l'Etat japonais peut créer et manipuler des intérêts parfois en créant lui-même ses propres alliés privés mais il peut aussi être infiltré par eux et il est impossible de dire a priori qui est le colonisateur et le colonisé dans un cas spécifique d'élaboration d'une politique. 


\section{BIBLIOGRAPHIE}

Policy Conflit and its Resolution within the Governmental System. E. Krauss Ed. University of Hawai Press, 1984.

Japan's Economy in war and Reconstruction. J.B. Cohen, University of Minnesota Press, 1949.

Modern Japanese Organisation and Decision Making. G.L. Curtis Editeur. Ezra Vogel University of California Press, 1975.

Gendai Nihon Seiji Keizai no Kozu T. Inoguchi, 1983. Toyo Keizai.

Between Power and Plenty. P. Katzenstein University of Wisconsin Press 1983.

Between MITI and the Market : Japanese Industrial Policy for High Technology. D. Okimoto Stanford University Press, 1989.

Governments, Markets and Growth : Financial Systems and the Politics of Industrial Change. J. Zysman-Cornell University Press, 1984. 


\section{SUMMARY}

The Japanese state is often perceived as the archetype of the strong state controlling the society in all its aspects.

The reality should be nuanced. Not only the state has never been an important commercial actor on the market but it has always been obliged to compose with private interests during the elaboration and enforcement of the legislation. Its involvement in the industrial policy was important in the development of the industrial basis but was never accepted without condition. The State influence results of a continuous negociation process with the private interest in which nobody can tell who is controlling or dominating the other. Moreover, both the state and the private concerns are heterogenous and must cope with diverging interests among themselves. Those interests have evolved over time and according to the industries considered.

A Japan Inc in which all parties share the same concern has never existed. Everybody fights for its own interest and tries to keep the profit and transfer the losses. It is difficult to talk of a consensual approach, but more of a kind of reciprocal acceptation of ever changing situation. On one hand, the result is a state never trusting completely the market but whose action is always reinforcing market structures because of the pervasive influence of private concerns and, on the other hand, private interests relying on the state and usingit to make regulations reinforcing their position on the market. 